\title{
Cryogenic imaging and spectroscopic study of electrochemically formed solid interphases - from nano to meso scale.
}

\author{
Y. Shirley Meng \\ University of California San Diego, La Jolla, California, United States
}

Our work demonstrated that the chemical and morphological information of Li metal can be largely preserved during cross-sectional TEM specimen preparation via cryo-FIB by mitigating the local heating damage and ion implantation, which has enabled the quantitative analysis of the impact of electrolyte on the nucleation and morphology of Li metal. Cryo-FIB has also shown potential to elucidate the structure and chemistry of $\mathrm{Li}$ metal/electrolyte interfaces when coupled with cryo-TEM. Now we have developed unique in situ and cryogenic analytic electron microscopy techniques to probe dynamic phase changes and interfaces of susceptible materials for electrochemical devices. Intrinsic chemical reactivity and beam sensitivity of Li metal and solid electrolyte interphase (SEI) impede mechanistic studies by electron microscopy. Solar cells with hybrid perovskite materials are also beam sensitive and their degradation issues need to be studied upon operation under the electrical bias. To study these materials, prerequisite techniques are required including cryogenic focused ion beam (cryo-FIB) and cryogenic transmission electron microscopy (cryo-TEM).

These efforts are based on the advances of the chip-based in situ methods of characterizing solid-state interfaces. Our recent work on Li/LiPON interphase through cryo-FIB and cryo-TEM elucidates the coexistence of several nanostructured species that embedded in an amorphous matrix, which can stabilize $\mathrm{Li}$ metal anode during the electrochemical reaction. Another work on perovskite materials under the electrical biasing via chip-based in situ holder has revealed for the first time the degradation mechanisms of perovskite solar cells through facet dependent amorphization.

It is now time for us to expand the range of materials systems and interfaces, and further capture the dynamic behavior of beam-sensitive functional materials in TEM through the combination of cryo-FIB and cryo-TEM techniques. It is important to realize that the cryogenic imaging and spectroscopy study of materials science is only at its infancy.

\section{References}

1. M.C. Kim, N. Ahn, D. Cheng, M. Xu, X. Pan, Y. Luo, D. P. Fenning, S. J. Kim, S.-Y. Ham, K. Jeong, M. Choi, Y. S. Meng, In-situ TEM observation of Perovskite Materials Amorphization under electrical bias, Advanced Energy Materials, under review

2. Cheng, T. A. Wynn, S. Wang, M. Zhang, S. Bai, H. Nguyen, R. Shimizu, C. Fang, B. Lu, X. Wang, Y. S. Meng, Unveiling the Li/LiPON Interface via Cryogenic Electron Microscopy, Joule, 2020.

3. X. Wang, G. Pawar, Y. Li, X. Ren, M. Zhang, B. Lu, A. Banerjee, P. Liu, E. J. Dufek, J. Zhang, J. Xiao, J. Liu, Y. S. Meng and B. Liaw, "Glassy Li metal anode for high-performance rechargeable Li batteries ", Nature Materials, 2020, 19, 1339-1345 